\title{
CPW-fed circularly-polarized antenna array with high front-to-back ratio and low-profile
}

https://doi.org/10.1515/phys-2018-0082

Received May 31, 2018; accepted July 6, 2018

\begin{abstract}
A novel design of a coplanar waveguide (CPW) feed antenna array with circular polarization (CP) and a high front-to-back ratio is described. The proposed $\mathrm{CP}$ array is achieved by using a compact CPW-slotline transition network etched in the ground plane. The measured results show that this kind of feeding method can improve the impedance bandwidth, as well as the axial ratio bandwidth of the CP antenna array and provide adequate gain. The proposed array can achieve a $6.08 \%$ impedance bandwidth and a $4.10 \%$ CP bandwidth. Details of the antenna design and experimental results are presented and discussed.
\end{abstract}

Keywords: low-profile antenna, coplanar waveguide, CPW-slotline transition, circular polarization, sequential feeding

PACS: 41.20.Jb, 84.40.Ba

\section{Introduction}

Circularly polarized (CP) antenna arrays with a single layer structure are very attractive in modern communication systems because of several inherent advantages, such as insensitivity to depolarization and reduced multi-path effects. However, these arrays usually suffer from narrow axial ratio (AR) bandwidths, and especially when the dielectric slab is thin, the performance of such antenna arrays will be greatly limited due to their low bandwidths [1]. To solve this problem, printed aperture antennas are commonly used since they can provide a wide CP bandwidth,

Yilin Liu: College of Electronics and Information Engineering, Sichuan University, No.24 South Section 1, Yihuan Road, Chengdu, China, 610065, People's Republic of China; E-mail: 543786751@qq.com

*Corresponding Author: Kama Huang: College of Electronics and Information Engineering, Sichuan University, No.24 South Section 1, Yihuan Road, Chengdu, China, 610065, People's Republic of China; E-mail: huangkamascu@21cn.com but they exhibit a low gain due to their bidirectional radiation properties [2]. Bidirectional radiation not only results in a low gain in forward radiation but also wastes half of the input power in the backward direction. The multilayer substrate technique is another common method to improve the $\mathrm{CP}$ bandwidth and provides directional radiation [3]. Unfortunately, this approach, which requires complicated antenna structures, will not only increase the profiles of microstrip arrays, but also increases the manufacturing costs.

A CPW-fed microstrip antenna, similar to an aperturecoupled microstrip antenna, has a simplified structure with only two metallic layers. The electromagnetic energy can be coupled from the slot to the radiating patch. Furthermore, due to the slot cut in the ground plane, the electromagnetic energy within the cavity between the microstrip patch and the ground plane will be less confined, which lowers the quality factor of the cavity and in turn leads to a higher bandwidth [4]. This kind of antenna can be easily applied onto the surface of aircraft without influencing their aerodynamic configuration [5, 6]. The CPWfed antenna also has several advantages over traditional microstrip antenna, such as easy integration with active and passive elements, high circuit density, low dispersion, low radiation loss, and avoidance of ground vias. However, CPW has been rarely applied to feed antenna arrays until now, with most research focusing mainly on linearly polarized slot antenna arrays and printed monopole arrays [7-9]. As mentioned above, these printed aperture antenna arrays usually have bad front-to-back ratio due to their bidirectional radiation properties. The previously reported CPW antenna arrays are based on a complex power divider [10, 11], which needs air-bridges and multilayer construction [12]. This kind of power divider will increase the total weight of the antenna array and complicate the design processing of the $\mathrm{CP}$ antenna array.

\section{Antenna design}

The geometry of the antenna is shown in Figure 1. An initial antenna back fed by a CPW-slotline network is pro- 
posed. The circular antenna patch is dual-fed by two slotlines with transverse ends. The resonance frequency depends mainly on the radius of the circular patch. By selecting suitable lengths, the two feeding slotlines can have a length difference of one quarter wavelength and excite two orthogonal current components with the same magnitude and a $90^{\circ}$ phase difference on the circular patch surface for $\mathrm{CP}$ radiation.

The antenna patch and the CPW-slotline feeding network are placed on opposite sides of the substrate. This kind of antenna structure maximizes the use of space and can provide a broader bandwidth without using multilayer construction [4]. The low-cost FR4 substrate, $\mathrm{h}=1 \mathrm{~mm}$ and $\mathrm{Er}=4.4$, is used in experiments. The widths of the strip and slot of the CPW are set to be $3 \mathrm{~mm}$ and $0.3 \mathrm{~mm}$ respectively to make sure that the characteristic impedance of the CPW is $50 \Omega$. For simplicity, the widths of all slotlines are also set at $0.3 \mathrm{~mm}$ and all antennas are simulated by the ANSYS HFSS 15.0 simulation software.

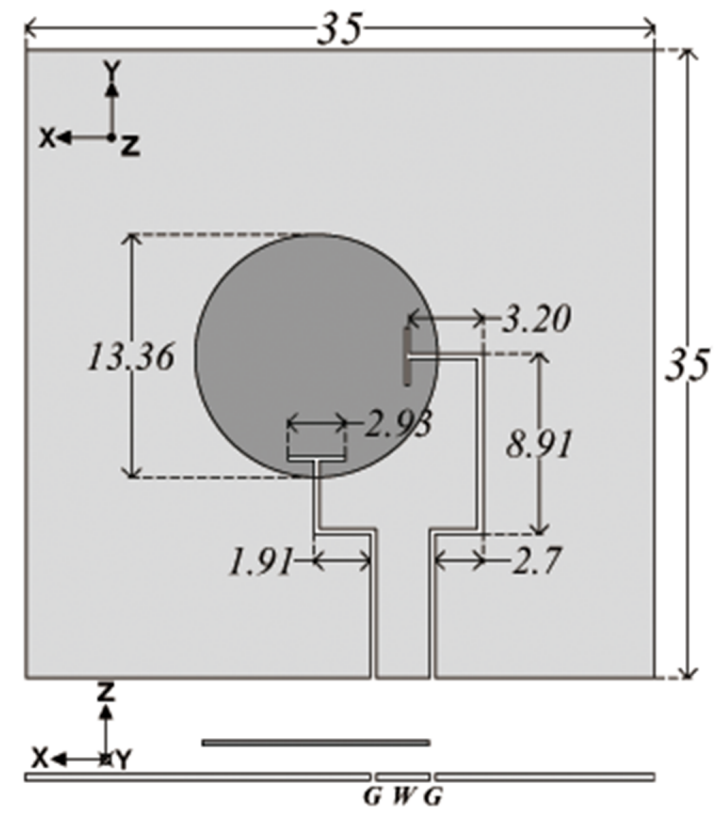

Figure 1: Configuration of the initial antenna

\section{Feeding network design}

Antenna arrays prevail in point-to-point [13] communications because they can provide a high gain and betterdirectionality. A $2 \times 2$ array based on the initial CPW-fed antenna is also proposed. Since a CPW feeding network poses a challenge in the design of CPW-fed arrays, the normal CPW feeding network needs CPW feeding lines with different characteristic impedances as well as air-bridge structures. Those requirements will enlarge the size of the feeding network, resulting in a difficulty to machine the antenna arrays. To reduce the complexity of the feeding network design, a feeding network based on a bridgeless CPW power splitter is thus proposed. The power splitter exploits the CPW odd mode, instead of suppressing it using air bridges, and it consists of one CPW-slotline T-junction and two slotline-CPW transition structures [14-16]. The CPWslotline T-junction transforms the input $\mathrm{CPW}$ even mode to a CPW odd mode, and the characteristic impedances of the CPW and theslotlines within the junction are set to be $50 \Omega$ and $100 \Omega$ respectively in order to match the structure. The layout of the slotline/CPW transition, shown in Figure 2, has one slotline input port and one CPW output, with the two remaining ports terminated with simple stubs. By optimizing the geometric shapes of the two stubs, the CPW odd mode will be transformed back to a CPW even mode and a realized power distribution.

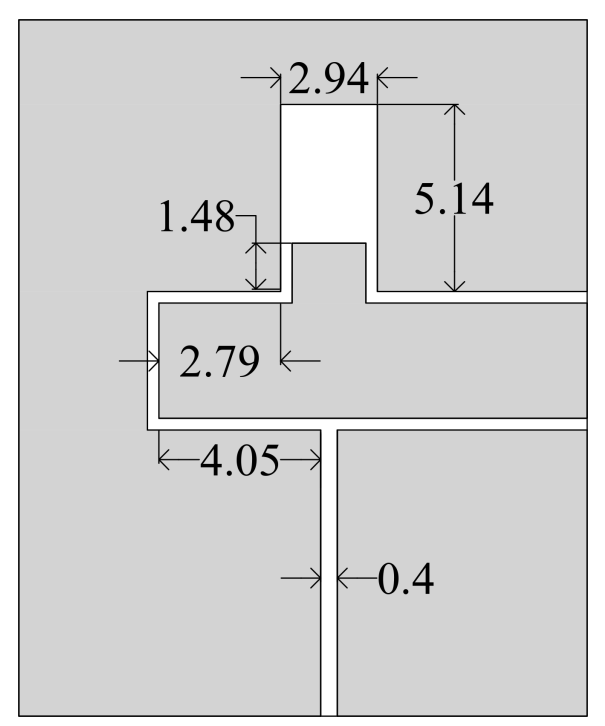

Figure 2: The Slotline-to-CPW transition

\section{Antenna array design}

By integrating the initial antenna with the CPW power splitter, a basic $1 \times 2$ array can be realized. Sequential rotation feeding is used to improve the array bandwidth, and the feeding network should be adjusted to provide proper spatial rotation and phase compensation for the left-hand 
circularly polarized (LHCP) $1 \times 2$ linear array. Each element of antenna 2, including the patch and its feeding structure, is physically rotated $90^{\circ}$ anticlockwise from the previous element. Furthermore, to ensure the in-phase radiation of the two patches after the rotation, the total length of the feeding slotline of patch 2 should be one quarter guided wavelength longer than that of patch 1 . By extending and rotating the basic $1 \times 2$ linear array by $180^{\circ}$, the proposed $2 \times 2$ array is constructed. The configuration of the proposed array is shown in Figure 3. As in the case of the $1 \times 2$ linear array design, a half-wavelength delay slotline is inserted before feeding patches 3 and 4 to ensure that the two linear arrays can still radiate in-phase. An important strength of the sequential rotation array is that the reflections caused by mismatched elements can cancel each other out at the corporate feed input terminal [17-19]. Therefore, the working bandwidth of $\mathrm{CP}$ antenna arrays can be improved. Figure 4 and Figure 5 denotes the simulated return loss and AR bandwidths of the initial antenna element and the $2 \times 2$ array. It can be observed that the return loss bandwidths remain almost the same but there is an improvement in the AR bandwidth with the $2 \times 2$ array.

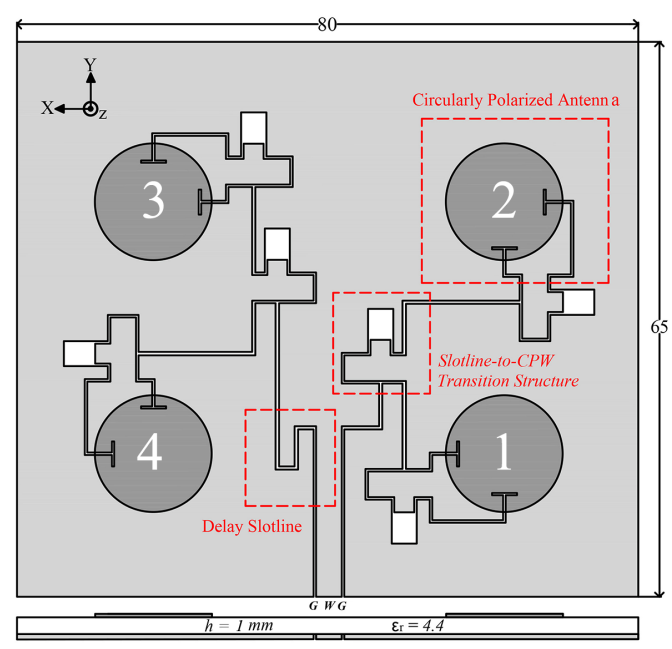

Figure 3: Configuration of the proposed array

\section{Results and discussion}

Figure 6 shows photographs of the $2 \times 2$ array. The measured and simulated return loss $\left(\mathrm{S}_{11}\right), \mathrm{AR}$ and gain of the $2 \times 2$ array are demonstrated in Figure 7 and Figure 8 .

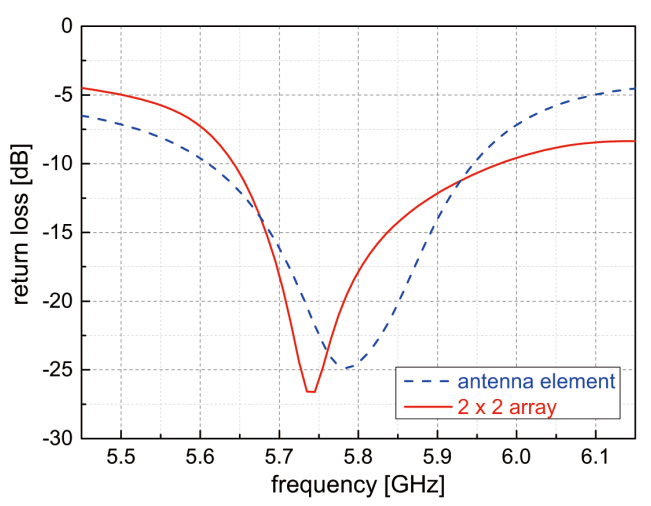

Figure 4: Simulated return loss of a CP antenna element and a $2 \times 2$ array

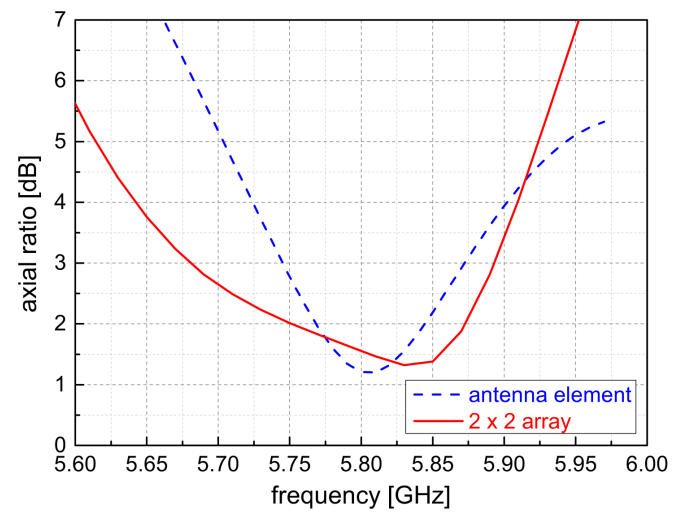

Figure 5: Simulated AR of CP antenna element and $2 \times 2$ array
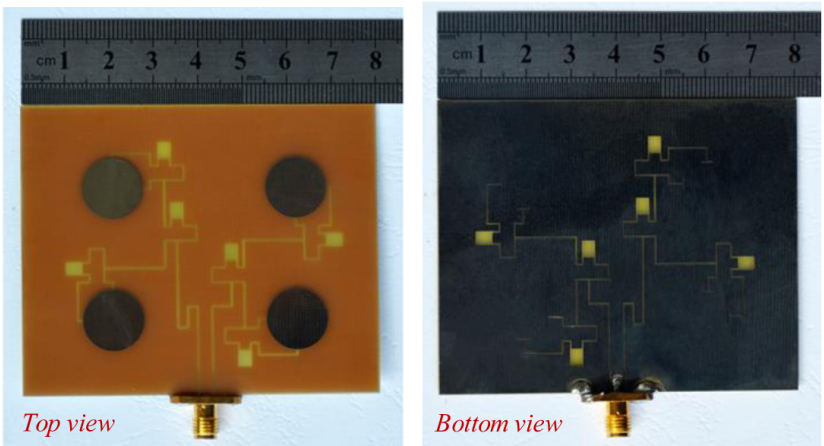

Figure 6: Photographs of the proposed $2 \times 2$ array

The discrepancy is probably caused by fabrication errors and by the welds between the SMA connector and the $\mathrm{CPW}$ structure. The measured 3- $\mathrm{dB}$ AR bandwidth of the $2 \times 2$ array is $4.10 \%$ higher from $5.660 \mathrm{GHz}$ to $5.897 \mathrm{GHz}$ and the measured impedance bandwidth is $6.08 \%$ higher from $5.605 \mathrm{GHz}$ to $5.957 \mathrm{GHz}$. The $\mathrm{S}_{11}$ is lower than $10 \mathrm{~dB}$ over the whole 3-dB AR bandwidth. The array gains in the boresight direction are measured by using the substitute method with a horn antenna of known gain, the maximum 


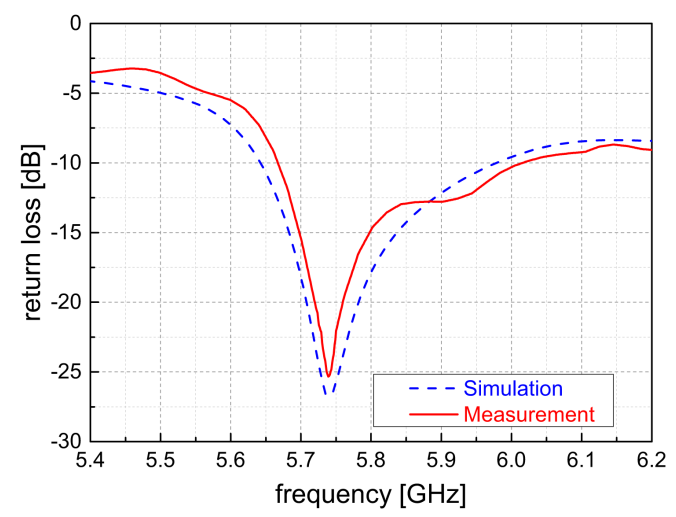

Figure 7: Measured and simulated return loss of the proposed array

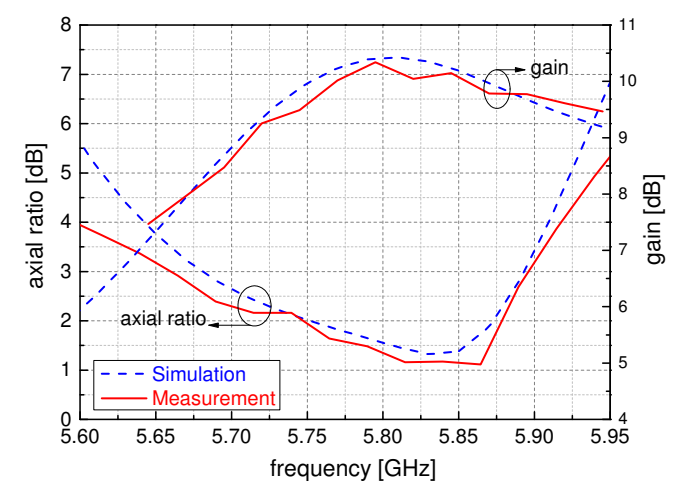

Figure 8: Measured and simulated gain and AR of the proposed array

measured gain is $10.25 \mathrm{~dB}$ in the $3-\mathrm{dB}$ AR bandwidth. The proposed design can be used as a unit block to form a larger array when a higher gain level is required.

Figures 9(a) and (b) depict the simulated and measured LHCP and RHCP radiation patterns of the proposed $2 \times 2$ array in the $x-z\left(p h i=0^{\circ}\right)$ and $y-z\left(p h i=90^{\circ}\right)$ planes at $5.8 \mathrm{GHz}$ respectively. Good left-hand CP radiation is observed and the main beams are somewhat shifted away from the $+z$-direction due to the asymmetry of the CPWslotline feeding network. Moreover, the front-to-back ratio of the proposed array is better than $18 \mathrm{~dB}$ in both the $x-z$ and $y-z$ planes.

The measured AR against azimuth angle of the initial antenna and the $2 \times 2$ plane array in the $x-z(p h i=0)$ and $y-z(p h i=90)$ planes at $5.8 \mathrm{GHz}$ are shown in Figure 10. It can be observed that the $\mathrm{CP}$ microwaves radiate to $+z$ directions.

In order to further evaluate the performance of the proposed design, a comparison between the $2 \times 2$ array and other similar arrays is shown in Table 1. It can be clearly seen that the proposed $2 \times 2$ array, with a simple configuration and a low profile, has an adequate gain within an

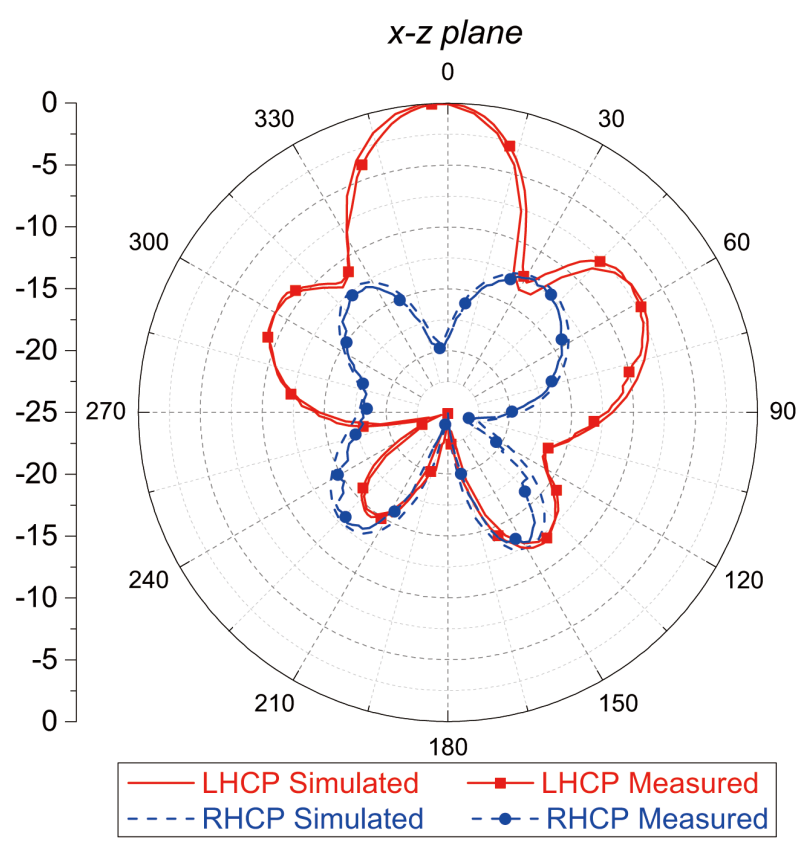

(a)

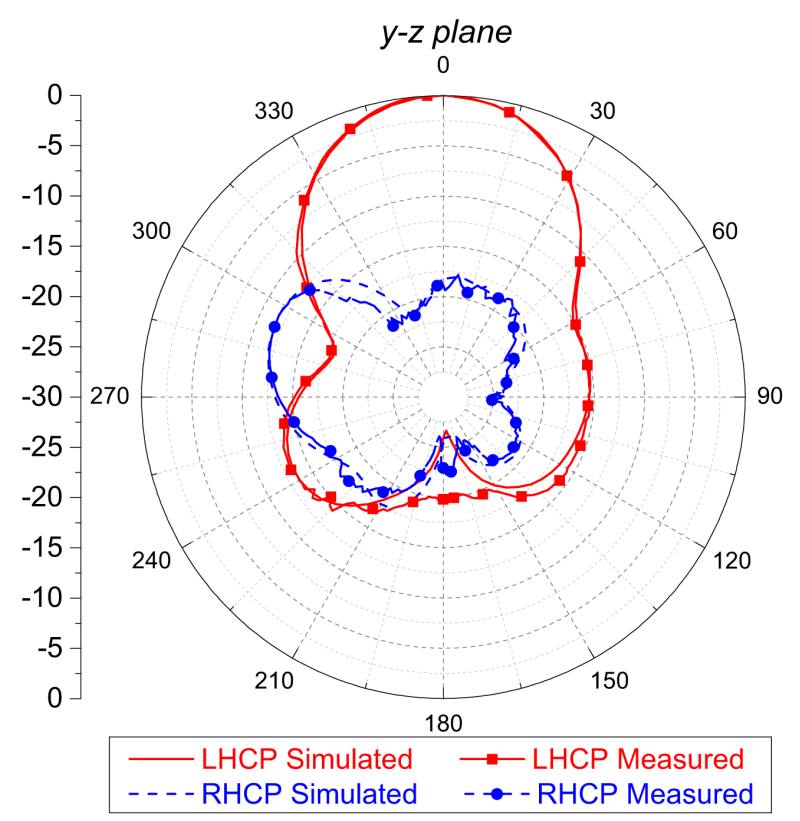

(b)

Figure 9: Measured and simulated radiation patterns of $2 \times 2$ array

adequate working bandwidth. Moreover, it is easy to fabricate owing to the simple structure and vialess single layer. 


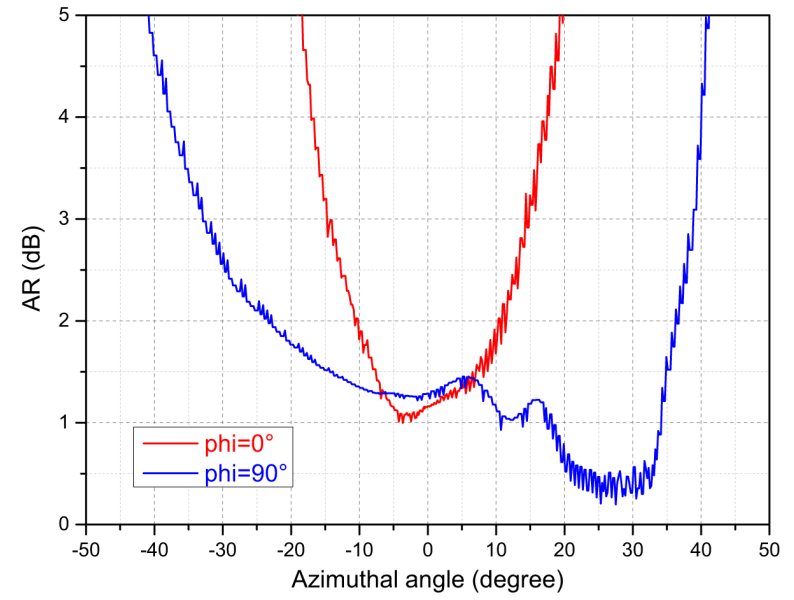

Figure 10: Measured AR against azimuth angle of the $2 \times 2$ array

Table 1: A comparison between the proposed array and other CP arrays

\begin{tabular}{ccccc}
\hline Reference & This work & {$[1]$} & {$[2]$} & {$[3]$} \\
\hline Frequency (GHz) & 5.8 & 2.5 & 6.0 & 5.8 \\
Structure & Single Layer & Single Layer & Single Layer & Multiple Layer \\
3 dB AR BW & $4.10 \%$ & $0.67 \%$ & $31.40 \%$ & $4.60 \%$ \\
Gain (dB) & 10.25 & 9 & 7.5 & 14 \\
Thickness (mm) & 1.0 & 3.18 & 0.8 & 2.85 \\
\hline
\end{tabular}

\section{Conclusion}

A novel $2 \times 2 \mathrm{CPW}$-slotline fed CP microstrip array with high front-to-back ratio has been proposed. The validity of this design has been established by comparison with the experimental results on return loss, axial ratios, radiation patterns and gains of a CP antenna element and a $2 \times 2$ array. The present designs have simple geometries, thin planar profiles and are relatively easier to be fabricated because of their single-layer structure. Additionally, due to the thin substrate and the reduced metal use, the proposed design is very lightweight, only about $10.13 \mathrm{~g}\left(0.211 \mathrm{~g} / \mathrm{cm}^{2}\right)$, which is a big advantage for aerospace applications.

Acknowledgement: The work is supported by the Youth Fund of Ministry of Education of China (Grant No. 6141A02033506) and the Defense Industrial Technology Development Program of China (Grant No. JCKY2016110B001).

\section{References}

[1] Zhang J.D., Zhu L., Liu N.W. et al., Dual-Band and Dual- Circularly Polarized Single-Layer Microstrip Array Based on MultiResonant Modes, IEEE Trans. Antennas Propag., 2017, 65, 14281433.
[2] Rafii V., Nourinia J., Ghobadi C. et al., Broadband Circularly Polarized Slot Antenna Array Using Sequentially Rotated Technique for C-Band Applications, Antennas Wireless Propagat., Lett. 2013, 12, 128-131.

[3] Fairouz M., Saed M.A., A Sequentially Rotated Circularly Polarized Microstrip Antenna Array with Reduced Mutual Coupling, Electromagnetics., 2016, 36, 422-433.

[4] Liu Y., Huang K., Yang Y. et al., A Low-Profile Lightweight Circularly Polarized Rectenna Array Based on Coplanar Waveguide, IEEE Antennas Wireless Propagat. Lett., 2018, 17, 1659-1663.

[5] Bing L., Ning Y., Research on the dynamic evolution behavior of group loitering air vehicles, Appl. Math. Nonlin. Sci., 2016, 1, 353-359.

[6] Martinez M.F., A survey on fractal dimension for fractal structures, Appl. Math. Nonlin. Sci., 2016, 1, 437-472.

[7] Chen X., Han L., Chen X. et al., A Wideband Coplanar Waveguide Antenna Array With Series Feed, IEEE Antennas Wireless Propagat. Lett., 2017, 16, 565-568.

[8] Xu L., Li L., Zhang W., Study and design of broadband bow-tie slot antenna fed with asymmetric CPW, IEEE Trans. Antennas Propag., 2015, 63, 760-765.

[9] Hua D., Qi S.S., Wu W. et al., CPW-fed printed antenna array with conical beam, IEEE Trans. Antennas Propag., 2016, 64, 10961110.

[10] Soliman E.A., Brebels S., Beyne E. et al., 22 and 44 arrays of annular slot antennas in MCM-D technology fed by coplanar CPW networks, IEE Proc.-Microw. Antennas Propag., 1999, 146, 335338.

[11] Soliman E.A. et al., Antenna arrays in MCM-D technology fed by coplanar CPW networks, IEEE Trans. Microwave Theory Tech., 2000, 48, 1065-1068.

[12] Soliman E.A. et al., Suppression of the parasitic modes in CPW discontinuities using MCM-D technology-application to a novel 3-dB power splitter, Appl. Math. Nonlin. Sci., 2016, 1, 44-50.

[13] Jian F., Dandan S., Complex network theory and its application research on P2P networks, IEEE Trans. Microwave Theory Tech., 1998, 46, 2426-2430.

[14] Nasr A.M.H., Safwat A.M.E., Air-bridge free coplanar waveguide power divider, European Microw. Conf., France, Sept. 2015, 474C477.

[15] Liu Y., Huang K., Luo X., Circularly Polarized Antenna Array Fed by Air-Bridge Free CPW-Slotline Network, Int. J. Antennas Propag., 2017, 2017, Art. no. 5230142.

[16] Nasr A.M.H., Safwat A.M.E., Multimode CoplanarWaveguide Cross Junction: Equivalent Circuit Model and Air-Bridge Free Applications, IEEE Trans. Microwave Theory Tech., 2017, 65, pp. 3753- 3760.

[17] Varum T., Matos J.N., Pinho P. et al., Nonuniform broadband circularly polarized antenna array for vehicular communications, IEEE Trans. Veh. Technol., 2016, 65, pp. 7219-7227.

[18] Rafii V., Nourinia J., Pourahmadazar J. et al., Circularly polarized circular slot antenna array using sequentially rotated feed network, Journal ofCommunication Engineering, 2012, 1, pp. 37-45.

[19] Liao S., Xue Q., Compact UHF three-element sequential rotation array antenna for satcom applications, IEEE Trans. Antennas Propag., 2017, 65, pp. 2328-2338 